\title{
Development of L-Type Fixation Equipment for Transporting Unit Modules
}

\author{
Kyoon-Tai Kim and Young-Hun Jun \\ Construction Policy Research Institute, Korea Institute of Civil Engineering and Building Technology (KICT), Goyang-Si 10223, \\ Gyeonggi-Do, Korea
}

\begin{abstract}
The unit modular technique is one in which unit modules are prefabricated in a factory and then constructed at a construction site. That is why an important process, transport of unit module, is added in this technique. However, it is not easy to transport a unit module that is high prefabrication ratio (prefab. ratio) (70 90\%), since the walls and interior and exterior materials installed in it can become broken when the unit module is fixed using general fixation tools, including ropes and fixing belts. When the external wall, windows, etc. are damaged during the transportation, the efficiency of unit modular method is deteriorated. Taking this into account, the purpose of this study is to develop an improved fixation device that is convenient for fixing a unit module with a high prefab. ratio to a vehicle and securing the safety while running. The L-type adapter block proposed in this study was evaluated the structural safety through simulation method. The result of the simulation is that it seems desirable to set the working load of the L-type adapter block as $15 \mathrm{kN}$ for Case 1 and $30 \mathrm{kN}$ for Case 2. Therefore, when the L-type adapter block is utilized under the conditions in which the same load is applied as in Case 2, it is expected that safety will be improved.
\end{abstract}

Key words: Unit modular technique, modular construction, fixation device, safety.

\section{Introduction}

\subsection{Background and Purpose}

In recent years, the limitations of the existing construction system, including stagnant productivity, excessive $\mathrm{CO}_{2}$ emissions and excessive construction wastage, have come the fore, and the unit modular housing method has been catching the industry's attention [1]. Unit modular systems, which maximize construction works performed in a factory, are attracting attention as an eco-friendly advanced construction method that can enable a revolutionary improvement in productivity while at the same time reducing carbon emissions and waste material [2].

In the unit modular technique, a cubic-type steel structure frame is produced in a factory, and then walls, windows and doors, diverse interior and exterior materials, facilities and electric wiring are

Corresponding author: Kyoon-Tai Kim, Ph.D., research fields: construction management/engineering and construction automation. E-mail: ktkim@kict.re.kr. equipped within it in the factory. The next, the unit module produced is delivered to a construction site and then assembled [3]. The unit modular technique has the advantages of easily enabling quality control, improving productivity and so on, because many construction works are completed in the factory. In addition, the steel structure frame can be easily disassembled, transferred and reused to minimize industrial waste. Therefore, the technique can be called eco-friendly method which reduce the environmental load [2].

Furthermore, the higher the prefabrication ratio (prefab. ratio) is, the more advantageous the unit modular technique is [4]. For this reason, to make the unit modular technique as an efficient method, and more eco-friendly, it is important to increase the prefab. ratio. To do this, construction work, such as attaching interior and exterior of wall, windows and so on, should be fabricated at the factory as many as possible. 
In the unit modular technique, an important process, transporting the unit module, is added because the unit module is prefabricated in a factory and constructed at a construction site [5]. If only the steel frames and floor slabs are manufactured in the factory, in which case the prefab. ratio is $50 \%$ or less and the unit module can be transported by fixing it to the vehicle using existing fixing methods, such as ropes or fixing belts. However, once walls, windows and even various interior/exterior materials have been installed in the factory, for a prefab. ratio of 70 90\% (Fig. 1), there is a risk of damaging the new walls and materials if they are fixed using existing methods [6, 7]. Also, workers may dislike performing fixing operations at times, because fixing a unit module that is shaped like a container box of about $3,000 \mathrm{~mm}$ in width, 6,000 $\mathrm{mm}$ in length and 3,000 $\mathrm{mm}$ in height, as shown in Fig. 1, using ropes or belts, is very inconvenient. It is, therefore, necessary to develop a new method for easily fixing and safely transporting unit modules with a high prefab. ratio on vehicles in order to maximize the performance and eco-friendliness of modular unit systems [8, 9].

Attempting to address this need, Park et al. [8] proposed an adapter block (Types A and B) and analyzed the adapter block's structural safety through a simulation. Also, Kim and Park [4] surveyed the adapter block's work convenience and test the adapter block's operation safety through an experiment. Through the tests, the adapter blocks (Types A and B) were found to have been improved in terms of securing safety ratio and work convenience.

This study aims to develop an improved fixation

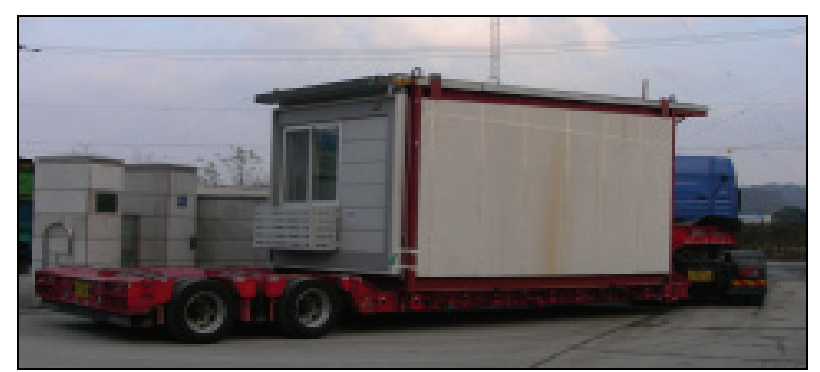

Fig. 1 Transport of a unit module with high prefab. ratio (70 90\%) by low-bed trailer. equipment for transporting unit modules, which is assembled in a factory more than $70 \%$. For this, an L-type fixation equipment is suggested in this study for conveniently fixing a unit module to a vehicle and securing safely.

Through the development of a transporting technology for the unit module, it is expected that the ultimate purpose of this study, to maximize the advantages of unit modular technologies, such as performance and eco-friendliness, can be reached.

\subsection{Method}

The experiment was conducted as follows:

(1) The existing fixation methods are examined, such as adapter block's design, simulations results and experiment data;

(2) An improved design of the fixation equipment is proposed;

(3) A simulation analysis is performed for reviewing the structural safety of the improved fixation equipment proposed.

\section{Status of Transporting Unit Modules}

\subsection{Fixation Method of a Unit Module During Transport}

At present, either 5-t trucks or 25-t air suspension low-bed trailers are used to transport modular units from a factory to a site in Korea. Because transporting cost, efficiency and safety can differ significantly depending on the freight vehicle, the type of freight vehicle is selected after taking the prefab. ratio, urgency of the work and the number of products to be transported by a unit modular company. The unit modules manufactured in a factory are loaded onto a freight vehicle using a fork lift or a gantry crane. To transport the unit module loaded on a vehicle, the unit module is fixed to the freight vehicle using chain, rope or fixing belt [10]. The owners of freight vehicles individually install fixing blocks on their vehicles, as shown in Fig. 2 to improve the convenience of the freight fixation work $[1,11]$. There are no such fixing 


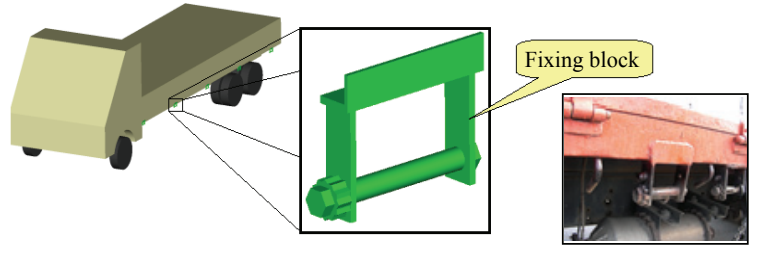

Fig. 2 Fixing blocks welded to a truck by owner.

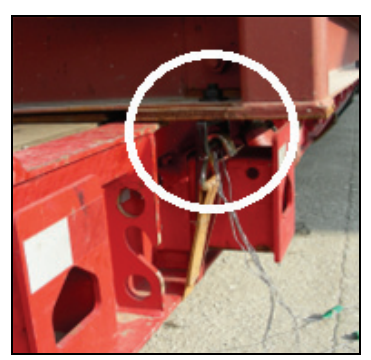

(a)

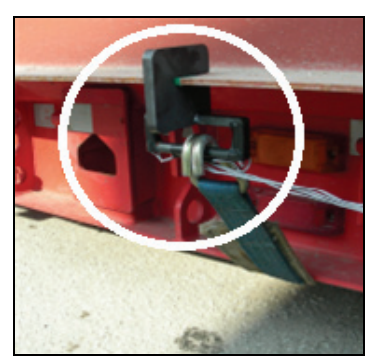

(b)

Fig. 3 Adapter blocks: (a) Type A; (b) Type B.

blocks on the vehicles when they are released from motor companies, but most of the truck owners individually install them by welding to enable ropes and fixing belts to be easily hung in Korea. Therefore, when the unit module is transported by conventional method, the unit module is fixed to the truck by fastening the rope or fixing belt, binding the unit modules to the fixing block [8].

\subsection{Developing History of Adapter Blocks}

\subsubsection{Design of the Adapter Blocks}

Adapter blocks (Types A and B) have been developed by the KICT (Korean Institute of Civil Engineering and Building Technology). Because it

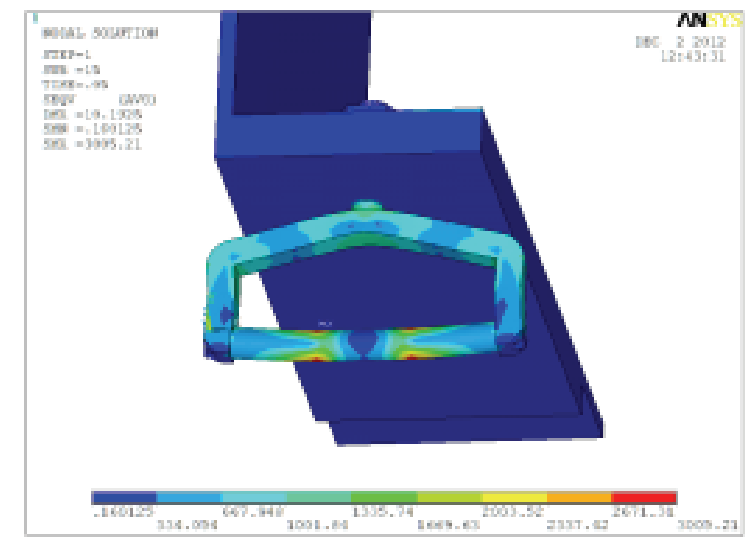

(a) uses the substructure of the unit module, it can connect the unit module and a freight vehicle without any interruption of walls, windows and doors and internal/external materials. In addition, the existing structures including the fixing block, the hole of the unit module and $\mathrm{C}$-channel are used to fix the unit module so that it has the advantage of enabling the existing vehicle and the unit module to be used without any additional changes. Therefore, it is possible to transport the unit module efficiently and eco-friendly without any damage which takes higher prefab. ratio [10]. Adapter block (Type A) is fixed on the hole located at the lower part of the multi-square column of the unit module using bolts and nuts. Adapter block (Type B) is fixed using a friction force by inserting at the lower part of the $\mathrm{C}$-channel of the unit module, as can be seen in Fig. 3. In sum, adapter block Type A is rather tedious to install but provides stable support, while adapter block Type B is easy to install but provides less support than block Type A [8].

\subsubsection{Structural Simulation of the Adapter Blocks}

A structural simulation was performed to check the safety before the mock-up test. The analysis factors of the adapter block, light-gauge steel and steel plate had eight joints, and each joint has solid 186 elements which has three degrees of freedom were used. Values were set as $206 \mathrm{GPa}$ for elastic coefficient, $1.45 \mathrm{GPa}$ for tangential modulus, $750 \mathrm{MPa}$ for yield strength

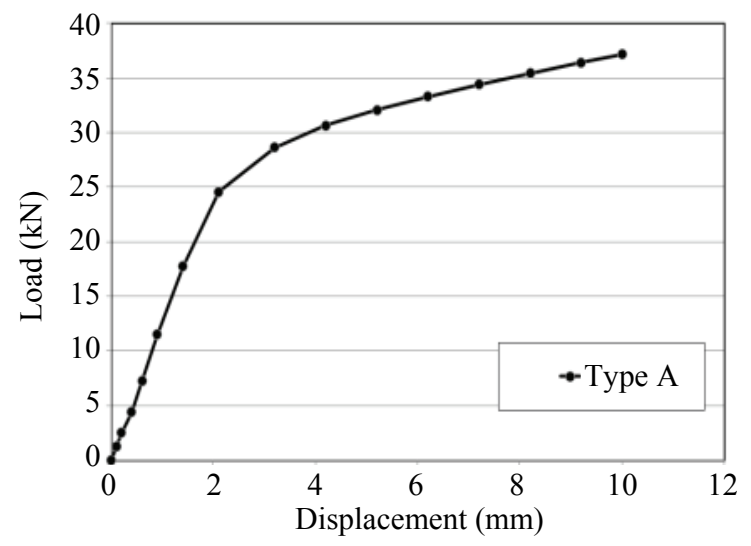

(b)

Fig. 4 Simulation results of adapter block (Type A) [7]: (a) stress distribution; (b) load-deflection analysis result. 


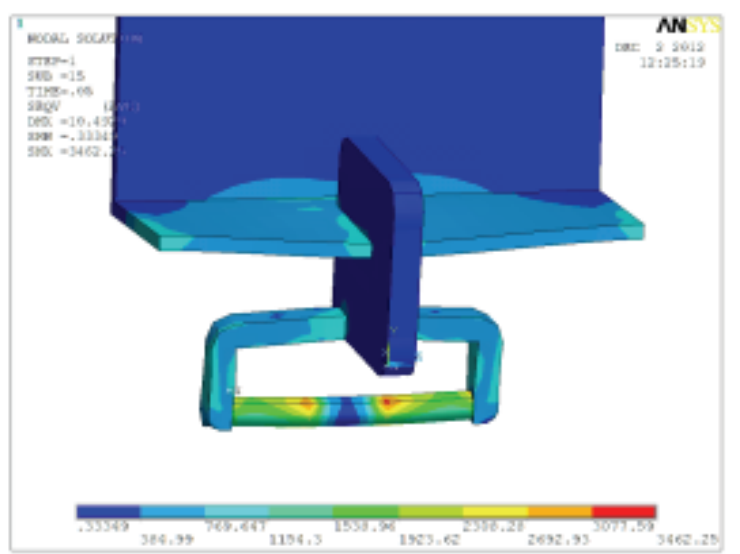

(a)

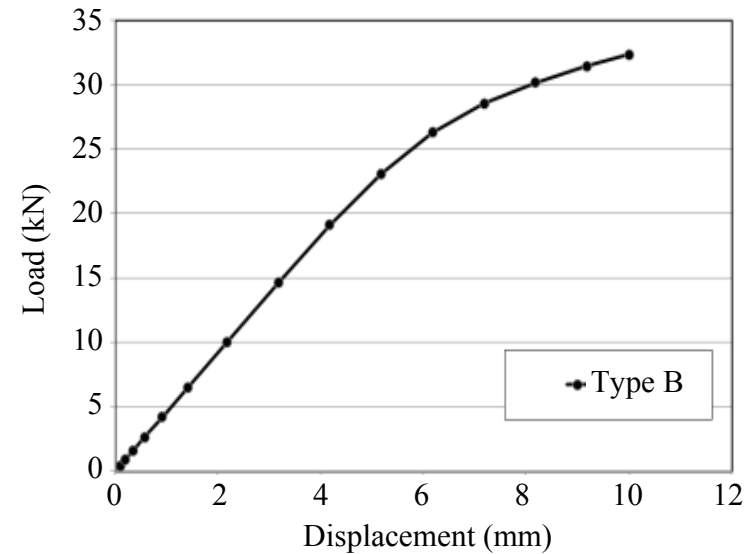

(b)

Fig. 5 Simulation results of adapter block (Type B) [7]: (a) stress distribution; (b) load-deflection analysis result.

and 0.3 for Poisson's ratio. In the modeling, the adapter blocks and the part of the unit module were built. ANSYS 13.0 was used to perform the modeling, creation of an element and finite element analysis of the adapter block without any additional use of the preprocessor. The results of the simulation are shown in Figs. 4 and 5. The stress load appeared at $24.6 \mathrm{kN}$ for adapter block Type $\mathrm{A}$ and at $28.5 \mathrm{kN}$ for adapter block Type B, respectively [8].

\subsubsection{Mock-Up Test of the Adapter Blocks}

The analysis of the four driving experimental runs revealed that a maximum load of $15 \mathrm{kN}$ was applied on adapter block Type A and a maximum load of $25 \mathrm{kN}$ was applied on adapter block Type B [4]. These loads were recorded at the points in the road test at which the low-bed trailer was driving through unstable sections of the test, such as stopping, restarting, passing over a speed bump or taking a turn at speed. The simulation results performed by Park et al. [8] predicted the yield load of adapter block Type A to be about $24.6 \mathrm{kN}$ and that for adapter block Type B as about $28.5 \mathrm{kN}$. As the maximum loads measured here were recorded when the vehicle was moving fairly rapidly through unstable sections of the test route, it seems likely that loads considerably below the yield load will be experienced by the adapter blocks when the vehicle is moving consistently at a reasonable speed throughout, as it would be the case in an actual modular unit delivery operation (Fig. 6).

\section{Design Improvement of the Adapter Block}

\subsection{Direction of Design Improvement}

Although field workers evaluated the adapter blocks to be much more convenient compared with the existing system of binding with rope, belt, etc., there were two things to be improved. In this study, we derived ideas to develop one type adapter block, because dealing with the two blocks, Types A and B, at once was considered rather tedious and inconvenient. We also try to secure a safety factor sufficient for the stress load to endure the maximum load because it was assessed that the safety factor of the adapter blocks was not secured in the test drives. Thus, when a truck runs on the unstable section at a relatively higher speed, it appeared that the adapter block Types A and B were rather vulnerable.

\subsection{Deduction of Improved Ideas}

In this study, an L-type adapter block, which looks like an L-shape viewing from the side, was proposed. This only one type of adapter block could connect a unit module to a vehicle using a hold in unit module. The adapter block had an extruding part and its center diameter was $14 \mathrm{~mm}$. The adapter block could be secured by pushing the extruding part of the adapter in the hole located at the lower part of the unit module as 


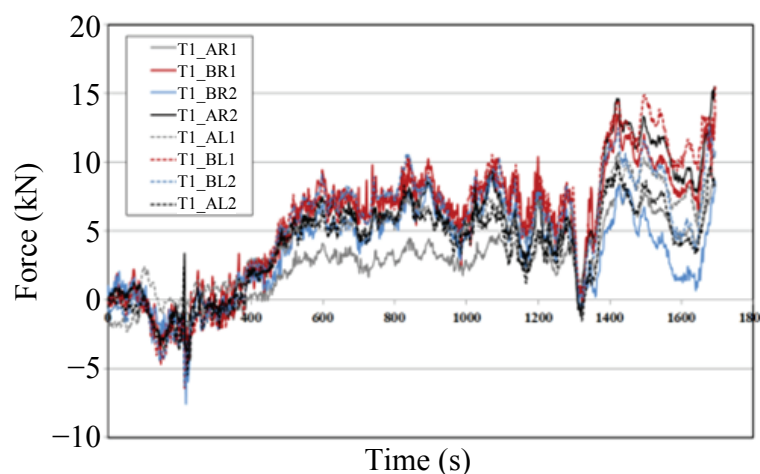

(a)

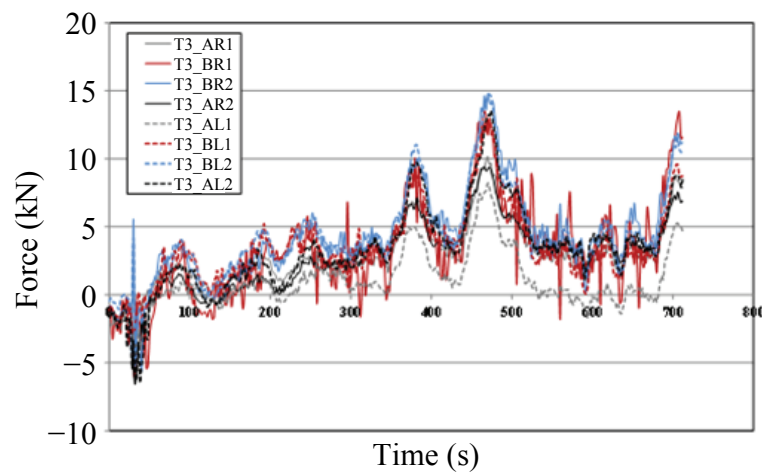

(c)

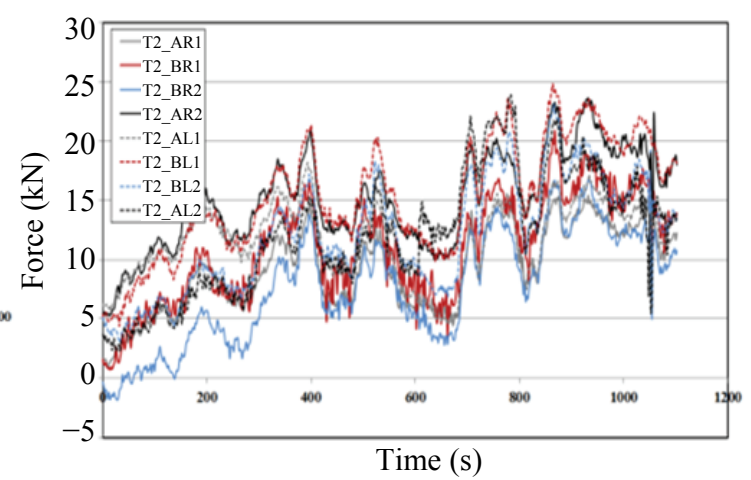

(b)

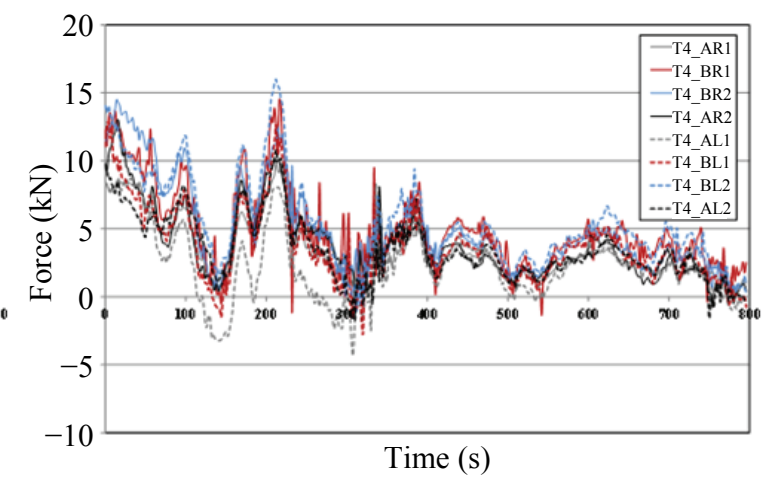

(d)

Fig. 6 Strain gauge readings for the driving experiment [3]: (a) Test 1; (b) Test 2; (c) Test 3; (d) Test 4.
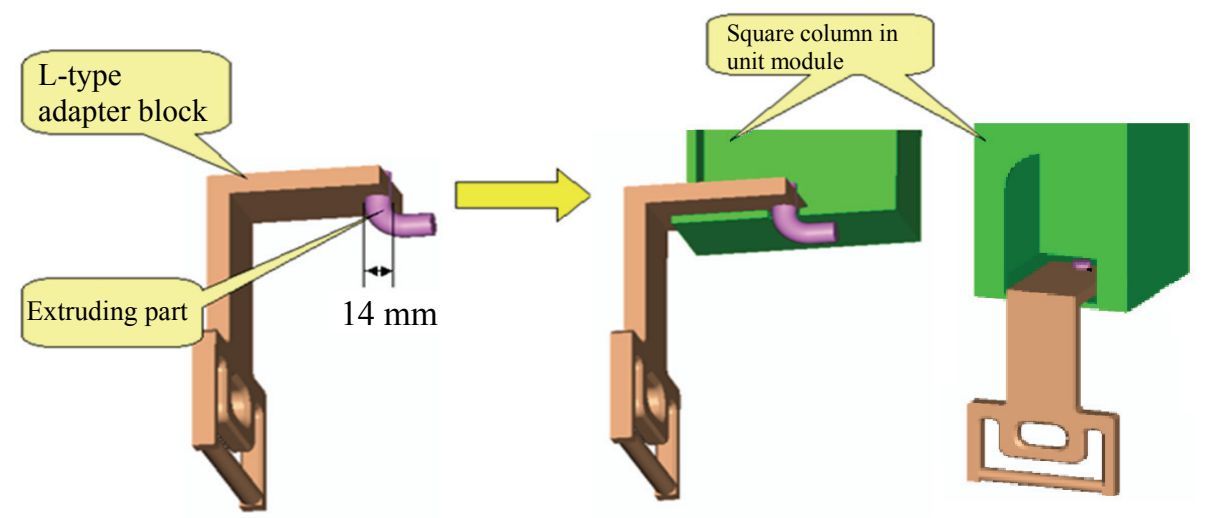

Fig. 7 Conceptual design of L-type adapter block.

shown in Fig. 7.

The shear strength requirements were expected to be met when the L-type adapter block was made of SS400 (Steel-Structure 400), like the existing adapter block. The diameter of the extruding part was $14 \mathrm{~mm}$, as described above, and the sectional area of the structure was:

$$
\pi r^{2}=3.14 \times 7 \times 7=153.9 \mathrm{~mm}^{2}
$$

The shear strength of SS400 was based on about $320 \mathrm{~N} / \mathrm{mm}^{2}$. At this time, the tensile strength was:

$153.9 \mathrm{~mm}^{2} \times 320 \mathrm{~N} / \mathrm{mm}^{2}=49,235 \mathrm{~N}=5,024 \mathrm{~kg}$ (2)

Therefore, it is expected to have about $5 \mathrm{t}$ of shear strength on one side during the transport. In addition, it should be noted that, after heat treatment, the shear strength increases due to the increased degree of hardness, however, the ductility decreases, making it more fragile [9]. 


\subsection{Fixation Method Using the L-Type Adapter Block}

The fixing process of the L-type adapter block in the hole located at the lower part of the unit module can be illustrated as shown in Fig. 8.

\section{Evaluation of the Improved Design}

\subsection{Outline of the Structural Simulation}

In this study, a simulation model was constructed of the L-type adapter block. For the efficiency of the analysis, a half model was used. A mid-section of the block restrained the $x$-direction, a plane part on which the modular structure met restrained the $y$-direction and $1 / 4$ of the diameter part of the modular structure restrained the $z$-direction. There were two load conditions, Case 1 and Case 2, to perform the analysis. In Case 1, the load was applied in the vertical direction, and in Case 2, the load was applied at a $45^{\circ}$ angle. In the two cases, it was hypothesized that the load was applied using a $50 \mathrm{~mm}$ long band. Therefore, the load was applied in the $25-\mathrm{mm}$ section at an equivalent distribution (Fig. 9).

\subsection{Direction of Design Improvement}

The structural simulation results of Cases 1 and 2

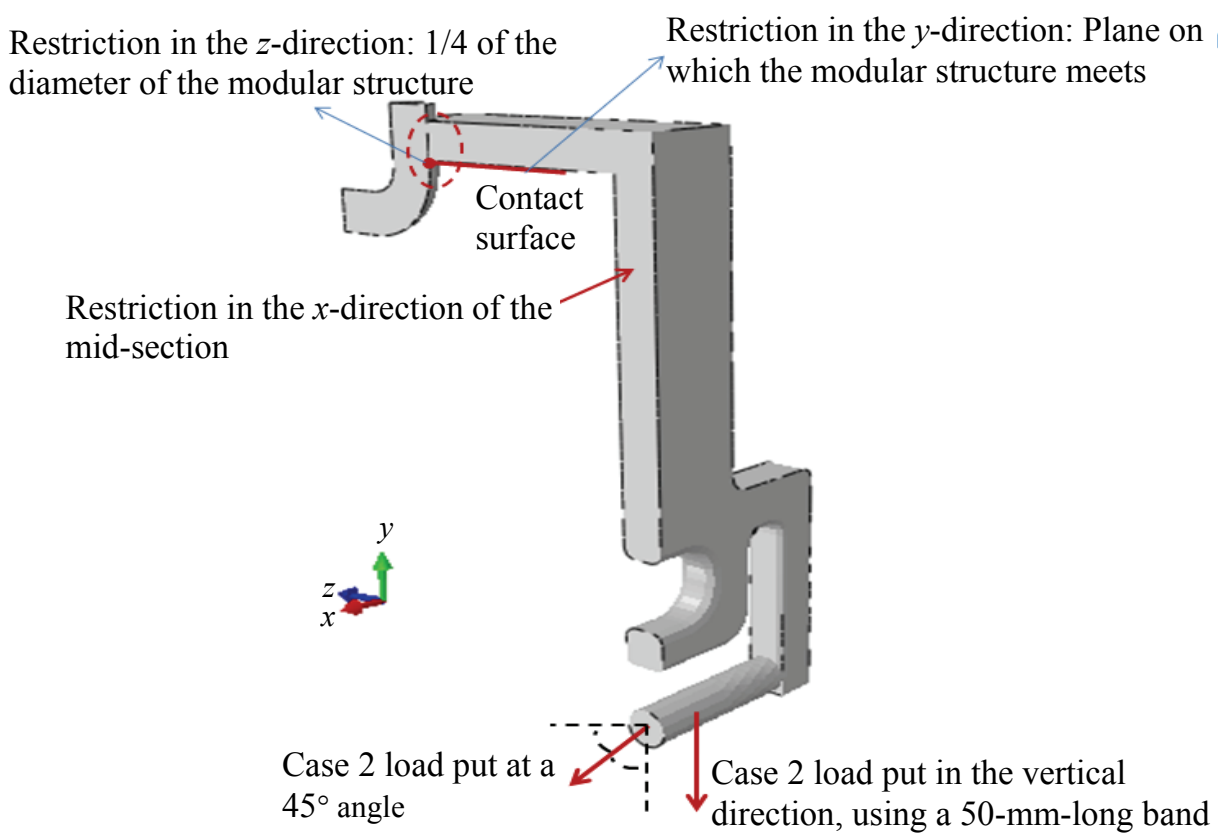

Restriction in the $y$-direction: Plane on are shown in Figs. 10 and 11, respectively. Figs. 10a and 11a show the deformed shapes observed in Cases 1 and 2, and Figs. 10b and 11b also show the stress distribution according to load in Cases 1 and 2, respectively. As you can see in these figures, the stress was greater at the extruding part where the load was directly applied than any other part. In addition, the stress distribution was observed to be more even in Case 2, where the load was applied at a $45^{\circ}$ angle, than in Case 1.

Through the simulation, we can summary the results that the L-type adapter block is that the nonlinearity became stronger from $15 \mathrm{kN}$ in Case 1 and from $30 \mathrm{kN}$ in Case 2, and the stiffness of the structure was deteriorated. In other words, it is desirable to set the load at $15 \mathrm{kN}$ for Case 1 and at $30 \mathrm{kN}$

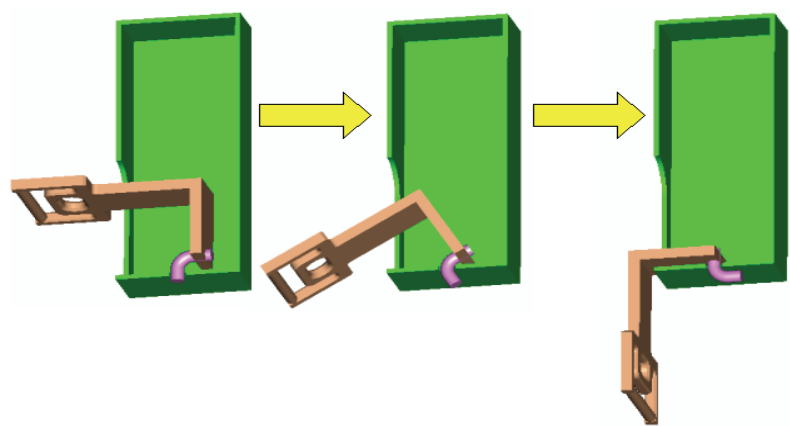

Fig. 8 Connection method of the L-type adapter block [8]. 


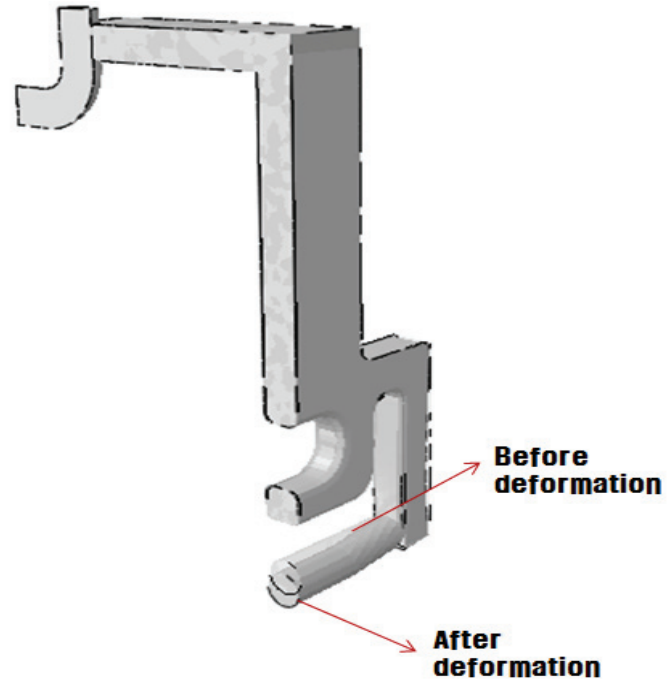

(a)
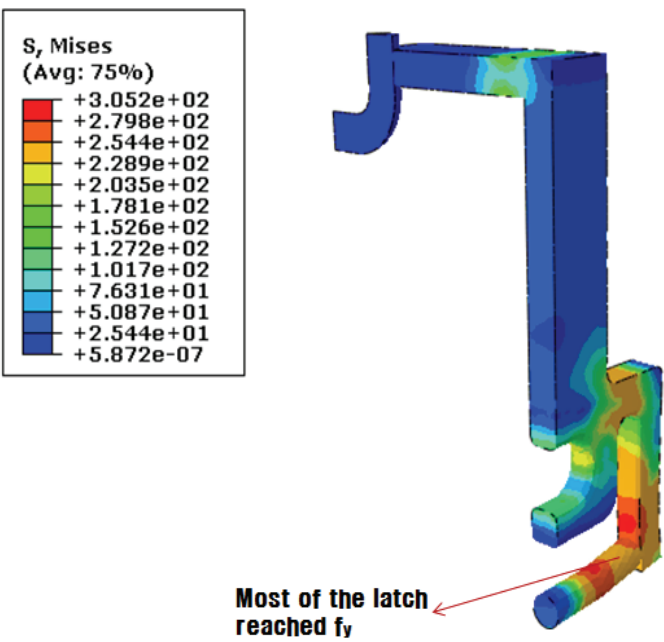

(b)

Fig. 10 Stress analysis of the L-type adapter block (Case 1): (a) deformed shape [11]; (b) von-mises stress state [12].

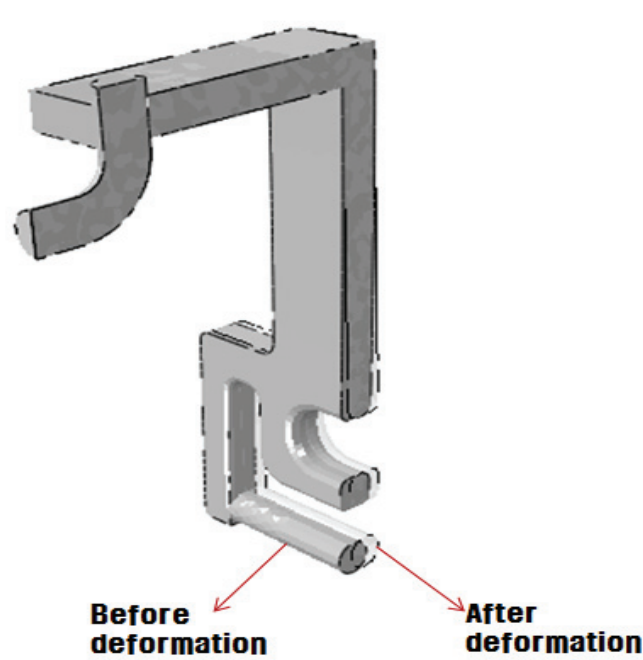

(a)
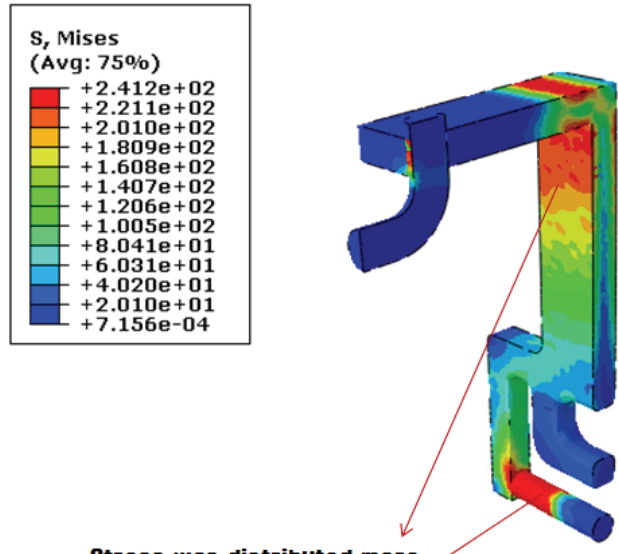

Stress was distributed evenly than in Case [1]

(b)

Fig. 11 Stress analysis of the L-type adapter block (Case 2): (a) deformed shape [11]; (b) von-mises stress state [12].

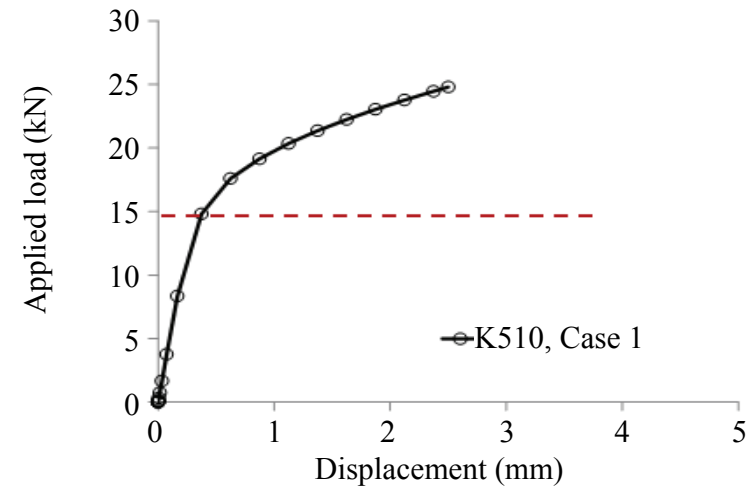

(a)

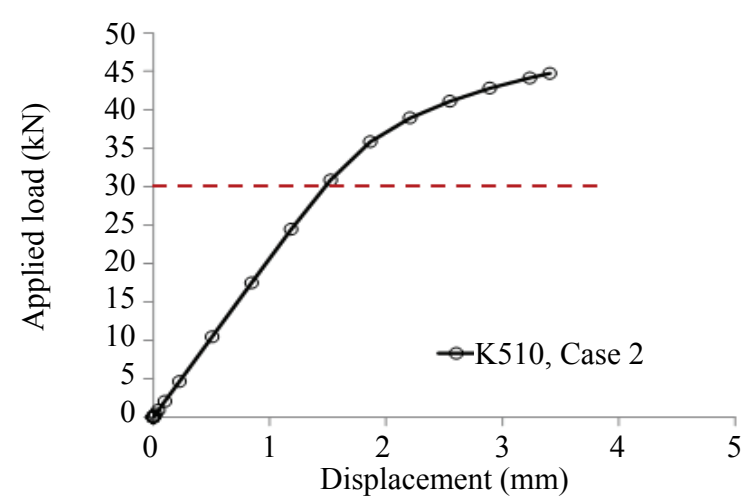

(b)

Fig. 12 Analysis results of the L-type adapter block [11]: (a) Case 1; (b) Case 2. 
for Case 2. Therefore, the L-type adapter block under the Case 2 condition is expected that safety will be more improved then under the Case 2 condition, in which the same load is applied. Here, it is noted that, when the load was applied at a $45^{\circ}$ angle, the working load of the fixed device was increased greatly (Fig. 12).

\section{Conclusions}

In the unit modular, construction technology which prefabricated unit modules in a factory and assembled them at a construction site should be added an important process, transporting the unit modules. If the external wall, windows, etc., attached to the unit module in the factory, are damaged during the transportation, the efficiency of unit modular method is deteriorated. Taking this into account, an improved adapter block, which fix the unit module to a truck and ensure no damage was caused to the external wall, is suggested in this study.

Attempting to address this need, an improved equipment, the L-type adapter block, was suggested in this study, and the structural safety was evaluated through simulation method. Summary of simulation results of the L-type adapter block is that the nonlinearity became stronger from $15 \mathrm{kN}$ in Case 1 and from $30 \mathrm{kN}$ in Case 2, and the stiffness of the structure was deteriorated. Therefore, it seems desirable to set the working load of the L-type adapter block as $15 \mathrm{kN}$ for Case 1 and as $30 \mathrm{kN}$ for Case 2. In other words, the L-type adapter block is utilized under the Case 2 condition, in which the same load is applied. However, it is difficult to determine that a sufficient safety factor was secured, and thus it should be improved further to secure the safety for the block.

\section{Acknowledgments}

This paper was supported by the KICT (Korean Institute of Civil Engineering and Building Technology) under the Strategic Research Project ("Construction Technology Development for One-day Housing"). This paper is the development result of the paper titled "Design and Analysis of Fixation Equipment for Transporting Prefabricated Housing Units" published in ICMDME 2015 conference (D233).

\section{References}

[1] Kim, K. T., Jun, Y. H., and Kim, T. Y. 2015. “A Study on the Supply Process of Unit Modular Housing through a Comparison of Cases." In Proceedings of the 6th International Conference on Construction Engineering and Project Management (ICCEPM 2015), 411-4.

[2] Kim, K. T., Chae, M. J., Park, N. C., and Park, S. Y. 2013. Development of Construction Technologies for One Day Housing. Technical Report for Korea Institute of Civil Engineering and Building Technology, Gyeonggi-Do, Korea.

[3] Lim, S. H., Park, K. S., Chae, C. U., and Kwon, B. M. 2007. "A Study on Factory Production of Modular Unit Housing Systems in Korea, United States, Japan, Europe." Journal of the Korean Housing Association 9 (2): 27-35.

[4] Kim, K. T., and Park, N. C. 2014. "Experimental Evaluation of Fixing Equipment for Improving the Efficiency of Modular Unit Systems." Journal of Building Construction and Planning Research 2: 244-54. doi: 10.4236/jbcpr.2014.24022.

[5] Kim, K. T., Kim, S., Park, N. C., and Lee, Y. R. 2014. "Development of Fixing Equipment for Transporting Modular Units." In Proceedings of the Korean Institute for Structural Maintenance and Inspection, 709-10.

[6] Park, S. Y., Kim, K. T., Park, N. C., and Chung, I. S. 2012. "Study of Improved Transportation Methods for Modular Units." In Proceedings of the Korean Institute of Building Construction (Industry), 243-4.

[7] Kim, K. T., Kim, S., Jun, Y. H., and Kim, T. Y. 2015. “A Comparative Analysis of Load Behavior of the Fixing Equipment for Transporting Unit Modules." In Proceedings of the Korean Institute of Building Construction, 246-7.

[8] Park, S. Y., Kim, K. T., and Park, N. C. 2013. "Development and Evaluation of Fixation Equipment for Transporting Modular Units." Journal of the Korean Institute of Building Construction 13 (6): 609-18.

[9] Kim, K. T., Jun, Y. H., Kim, S., and Park, N. C. 2015. "Design and Analysis of Fixation Equipment for Transporting Prefabricated Housing Units." In Proceedings of the 2015 4th International Conference on Machine Design and Manufacturing Engineering, 233.

[10] Kim, K. T., Lee, Y. H., Lee, D. H., and Kim, C. H. 2011. 
Development of Construction Technologies for One Day Housing. Technical report for Korea Institute of Civil Engineering and Building Technology, Gyeonggi-Do, Korea.

[11] Park, N. C., Kim, S., and Kim, K. T. 2015. "The Development of Fixing Equipment of the Unit Module Using the Probability Distribution of Transporting Load."
Journal of the Korea Academia-Industrial Cooperation Society 16 (6): 4267-75.

[12] Kim, K. T., Chae, M. J., Park, N. C., and Lee, Y. L. 2014. Development of Construction Technologies for One Day Housing. Technical report for Korea Institute of Civil Engineering and Building Technology, Gyeonggi-Do, Korea. 